\title{
Extracting Chemistry - Property Relationships by Mining Atom Probe Evaporation Events
}

\author{
Scott Broderick ${ }^{1}$ and Krishna Rajan ${ }^{1}$
}

1. Department of Materials Design and Innovation, University at Buffalo - State University of New York, Buffalo, NY USA

Atom probe tomography (APT) characterization of Ni-Co-Al-Ti alloys was performed and then assessed via a data mining approach in order to connect phase chemistry, degradation mechanism and bonding. The compositions of the alloys were varied to explore how the degradation of the material under extreme field changed not only as the alloy composition changed but also as the phase chemistries changed. APT gives incredible resolution of concentration profiles; however, this presents an issue when comparing between alloys which have systematic changes in terms of multicomponent chemistry. The purpose of this study is to explore the apparent influences of the neighboring atomic environment on the pair-wise interactions between atoms.

The alloys formed two phases: a $\gamma$ phase occupied by the Al and Ti atoms and a $\gamma$ ' phase occupied by Co atoms. Ni preferred the $\gamma$ phase, but also increasingly occupied the $\gamma^{\prime}$ phase with increasing Ni content. Therefore, tracking the evaporation of $\mathrm{Ni}$ atoms, as well as other atoms relative to $\mathrm{Ni}$, correlates degradation mechanism with chemistry. Further, in terms of site occupancy, Ni and Co share a site, while $\mathrm{Al}$ and $\mathrm{Ti}$ share the other site. We explore if different alloying chemistries influence the evaporation events of site occupancies and if ion evaporations are sensitive to site occupancy. To measure the field evaporation, we develop ion evaporation maps corresponding with each composition (Figure 1). This is done by mapping the mass-to-charge ratio of each atom in the dimer against the other atom in the dimer, where the higher number of incidents of a particular dimer chemistry corresponds to a higher relative bond strength between those two ions [1]. Therefore, by tracking the change in Ni hits in the ion evaporation map with changing composition, we assess the relative bond strength differences as a function of phase chemistry. To quantify these differences, we apply a dimensionality reduction approach considering the ion evaporation maps as a series of spectra. In order to gain useful quantitative data concerning the pair-wise interaction between different elements, cross-sections of the ion evaporation map (which has dimensions of $\mathrm{m} / \mathrm{n} 1, \mathrm{~m} / \mathrm{n} 2$, and number of incidents) can be extracted for elements of interest by considering a single $\mathrm{m} / \mathrm{n} 1$ value.

We have previously applied a dimensionality reduction to APT data for more accurate chemical measure of a phase [2]. Here we advance this to relating bonding and chemistry. The dimensionally reduced space captures the information of millions of measures into a 2D mapping, with the points corresponding to overall material chemistry, and the chemistry of ions evaporating with each chemical component (Figure 2). This integration of data mappings, new forms of describing atom probe data, and statistical learning analyses provides a methodology to characterize the complex chemical interactions which are otherwise buried in the large atom probe data space. This type of quantitative analysis is necessary when assessing chemical profiles in these systems due to potential differences in apparent concentration levels. This approach provides a normalization mean which allows us to compare evaporation events, which is very challenging to rigorously perform otherwise. For example, we show that preferential evaporation of elements with a given site occupancy changes as the overall chemistry changes, which is otherwise not notable merely by looking at the raw data [3]. 


\section{References:}

[1] J. Peralta, S. Broderick, K. Rajan. "Mapping Energetics of Atom Probe Evaporation Events through First Principles Calculations." Ultramicroscopy. 132, 143-151 (2013)

[2] S.R. Broderick, A. Bryden, S.K. Suram, K. Rajan. "Data Mining for Isotope Discrimination in Atom Probe Tomography." Ultramicroscopy. 132, 121-128 (2013)

[3] We acknowledge support from the Air Force Office of Scientific Research (AFOSR) under grant number FA9550-11-1-0158 and from the National Science Foundation (NSF) under grant number DMR-1307811.
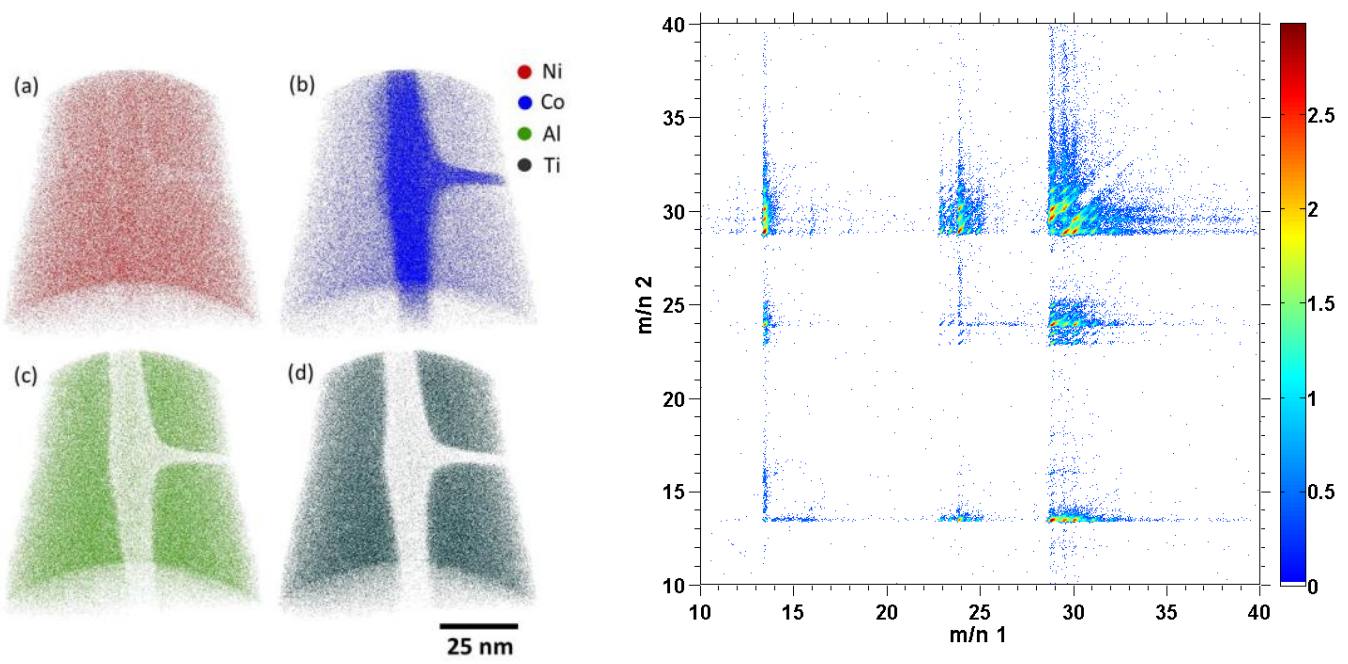

Figure 1. 3D Spatial position and ion evaporation map for a given chemistry. Such pairs are present for five different compositions of Ni-Co-Al-Ti. The spatial representation provides phase chemistries, while the ion evaporation is converted to an input for analysis representing relative bond strengths.
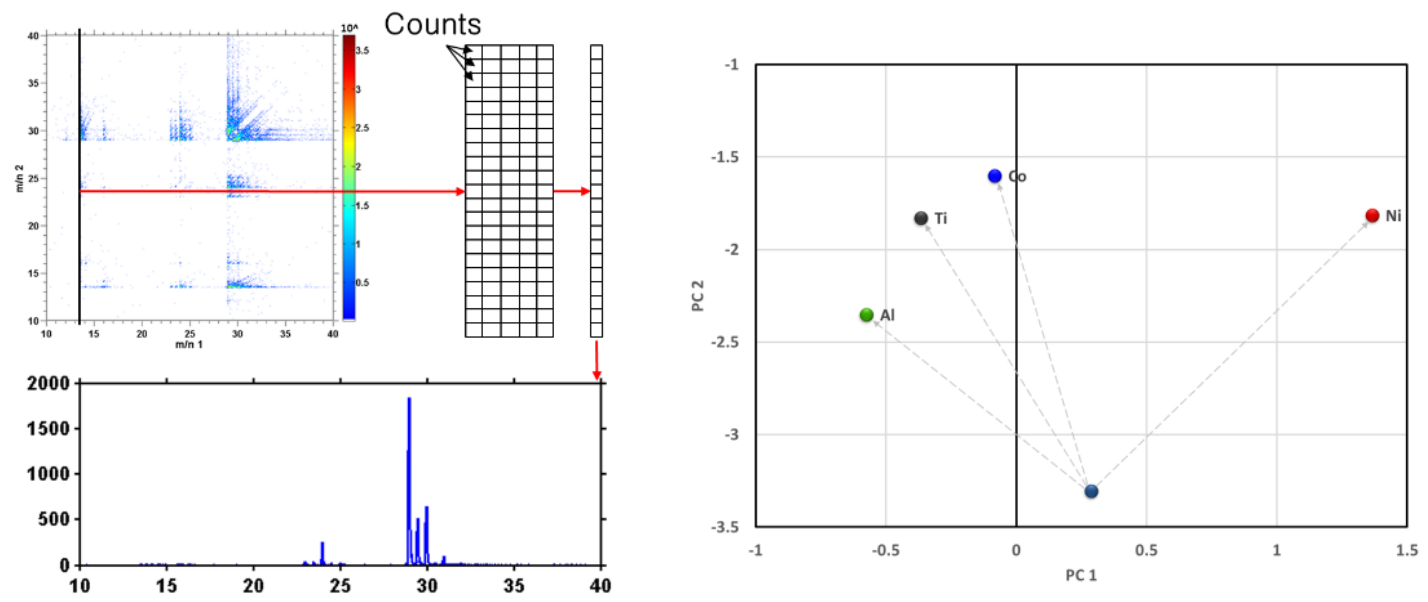

Figure 2. Method for developing spectra corresponding to particular chemistries (shown is concentration evaporating as dimer with $\mathrm{Al}$ ). This data processing provides a new representation of atom probe data, which is a suitable input for statistical learning analyses (right). The trajectories are compared for every composition, in order to identify the relationship between the phase chemistry / site occupancy and the relative bond strengths. 